\title{
Recent Developments in ab initio Thermodynamics
}

\author{
D. ALFÈ, ${ }^{1}$ G. A. DE WIJS, ${ }^{2}$ G. KRESSE, ${ }^{3}$ M. J. GILLAN ${ }^{4,5}$ \\ ${ }^{1}$ Geological Sciences Department, University College London, Gower Street, London WC1E 6BT, \\ United Kingdom \\ ${ }^{2}$ Electronic Structure of Materials, Research Institute for Materials, Toernooiveld 1, NL-6525 ED \\ Nijmegen, Netherlands \\ ${ }^{3}$ Theoretical Physics Institute, Vienna Technical University, Wiedner Hauptstr. 8-10, A-1040, Austria \\ ${ }^{4}$ Physics and Astronomy Department, University College London, Gower Street, London WC1E 6BT, \\ United Kingdom \\ ${ }^{5}$ DCI, CLRC Daresbury Laboratory, Warrington WA4 4AD, United Kingdom
}

Received 10 February 1999; accepted 1 September 1999

\begin{abstract}
It has recently become possible to calculate the free energy and other thermodynamic functions of solids and liquids using density functional theory to treat the quantum mechanics of the electrons. We present the main ideas that have made this possible, emphasizing the key role of thermodynamic integration and the importance of well-adapted reference systems in the computation of the free energy. We show how ab initio methods have been used to calculate the melting and other phase-equilibrium properties of simple materials, and the thermal-equilibrium concentrations of point defects in crystals. We point out the possibility of adapting techniques that are already available in order to calculate chemical potentials, solubilities, equilibrium constants, and other thermodynamic functions that are important in physical chemistry. (C) 2000 John Wiley \& Sons, Inc. Int J Quant Chem 77: 871-879, 2000
\end{abstract}

Key words: ab initio; free energy; statistical mechanics; density functional theory; molecular dynamics

Correspondence to: M. J. Gillan.

Contract grant sponsor: NERC.

Contract grant number: GST/O2/1454.

Contract grant sponsor: EPSRC.

Contract grant numbers: GR/L08946 and GR/L38592.

Contract grant sponsors: GEC; U.K. Car-Parrinello Consortium.

Contract grant sponsor: Minerals Physics Consortium.

Contract grant number: GST/O2/1002. 


\section{Introduction}

$\mathbf{T}$ heoretical chemistry rests on the twin pillars of quantum mechanics and statistical mechanics, and it has long been clear that thermal equilibrium properties should be computable by ab initio methods. To obtain thermodynamic functions, all that is needed in principle is a quantum calculation of the energy eigenvalues $E_{n}$, which are then used in the standard formula for the partition function:

$$
Z=\sum_{n} \exp \left(-\beta E_{n}\right),
$$

where $\beta=1 / k_{B} T$. This then gives the Helmholtz free energy $F=-k_{B} T \ln Z$ and hence all other thermodynamic functions. For simple molecules this can clearly be done so that the ab initio calculation of the specific heat of a dilute gas, for example, is perfectly feasible. It is equally clear that this direct approach will not generally work in condensed phases. The ab initio calculation of the melting temperature of a material, for example, is not going to be done by computing its energy eigenvalues $E_{n}$.

In spite of the obvious difficulties, the ab initio calculation of melting properties has recently become feasible [1, 2]. Indeed, all the essential ab initio concepts are now at hand to calculate a wide range of condensed-phase properties, including solubilities, phase diagrams, thermal-equilibrium concentrations of lattice defects [3-6], free energies of surface adsorption, and equilibrium constants. The aims of this study are to summarize the key ideas that are making this possible and to give practical examples of ab initio thermodynamic calculations.

\section{Key Ideas}

In many condensed-phase problems, two major simplifications can be made. First, the electrons can be assumed to be in the ground state. Invoking the Born-Oppenheimer principle, we can say that if the $N$ nuclei in the system are found at positions $\mathbf{R}_{1}, \ldots, \mathbf{R}_{N}$, then the total energy (excluding nuclear kinetic energy) is $U\left(\mathbf{R}_{1}, \ldots, \mathbf{R}_{N}\right)$ : the total groundstate energy calculated with the nuclei at these positions. Second, the nuclei can often be treated as classical particles so that we can use the classical ap- proximation for the free energy:

$$
\begin{aligned}
F=-k_{B} T \ln \left\{\frac{1}{N ! \Lambda^{3 N}}\right. & \int d \mathbf{R}_{1} \cdots d \mathbf{R}_{N} \\
& \left.\times \exp \left[-\beta U\left(\mathbf{R}_{1}, \ldots, \mathbf{R}_{N}\right)\right]\right\},
\end{aligned}
$$

where $\Lambda$ is the thermal wavelength, given by $h /\left(2 \pi m k_{B} T\right)^{1 / 2}$ (we assume that there is only one kind of atom, whose mass is $m$ ). With these simplifications, the problem reduces to (a) the ab initio calculation of $U$ and (b) the evaluation of the integral over configuration space.

We want to stress that we are discussing here only methods in which $U$ is calculated ab initio for every set of positions $\left\{\mathbf{R}_{i}\right\}$ involved in computing the integral over configuration space. We distinguish this from approaches in which the total energy is represented by some kind of parameterized model. Even though the parameters in such a model might have been determined by fitting to $a b$ initio calculations, this is not what we mean here by "ab initio thermodynamics."

In order to deliver what is needed, the ab initio techniques must be capable of treating systems of many atoms and of exploring large regions of configuration space. There is only one effective way to do this at present: density functional theory (DFT) combined with the pseudopotential approach. DFT is too well known to need detailed explanation here. (For reviews, see, e.g. [7-9].) We recall its central principle, which is that the total ground-state energy of a system of electrons acted on by a potential (in practice, the electrostatic potential due to the nuclei) is uniquely determined by the electronic density distribution $n(\mathbf{r})[10,11]$. All the approximations are concentrated in the exchange-correlation component $E_{\mathrm{xc}}$ of the total energy. In the early days, the local density approximation (LDA) was always used for $E_{\mathrm{xc}}[10,11]$; this assumes that there is a density of exchange-correlation energy that is related to the local density $n(\mathbf{r})$ in exactly the same way as in a uniform electron gas. Nowadays, some form of generalized gradient approximation (GGA) is frequently used, and improved GGAs are continually being developed [12-14]. DFT is highly competitive in accuracy with other quantum chemistry methods and is widely used for calculating the ground-state energetics of both molecules and condensed-phase systems.

In the pseudopotential approach [15-20], the electrons are divided into two classes: core electrons and valence electrons. The core electrons are assumed to be in exactly the same state as in the 
free atom. The true interaction potential between valence electrons and atomic cores is replaced by an $\mathrm{ab}$ initio pseudopotential that has almost exactly the same scattering properties. The key property of the pseudopotential is that in the core region the resulting "pseudo-wave-functions" lack the rapid oscillations possessed by the true wave functions. Because of their smoothness, the pseudo-wave-functions are easy to represent in terms of mathematically simple basis functions, and the normal procedure is to use a basis of free-electron wave functions, or plane waves. In this scheme, it is natural, and usual, to employ periodic boundary conditions so that the extended system is represented as a collection of atomic cores and electrons in a primary cell, which is surrounded by periodic images of itself.

This DFT/pseudopotential/plane-wave method, first formulated by Car and Parrinello [21] as a way of performing ab initio molecular dynamics simulations on condensed matter, is now in widespread use for investigating both solids and liquids [22-25]. For given nuclear positions $\left\{\mathbf{R}_{i}\right\}$, the selfconsistent ground state and its energy $U\left(\mathbf{R}_{1}, \ldots, \mathbf{R}_{N}\right)$ are determined by minimizing the total energy functional with respect to the plane-wave coefficients. The calculated forces on the nuclei are then used with Newton's equation of motion to generate the trajectories $\mathbf{R}_{i}(t)$ of all the nuclei. To achieve this, time is discretized into finite steps, and the selfconsistent ground state and the forces are recomputed at each step.

There are several DFT/pseudopotential/planewave codes in common use. A review of some of the key technical concepts underpinning these codes is given in Ref. [22], although we note that there have been considerable developments since that review was written. Our work on the melting of $\mathrm{Al}$ was performed with the VASP code [23, 24], which is particularly stable and robust for metallic systems. We shall see below that high precision and excellent robustness are critically important in free energy calculations. Technical aspects such as k-point sampling and plane-wave cut-off, which are explained in the reviews [22-24], must also be under strict control if useful results are to be obtained.

Even with an accurate ab initio energy function $U\left(\mathbf{R}_{1}, \ldots, \mathbf{R}_{N}\right)$, the calculation of the free energy presents a major challenge, except in one case: the harmonic system. For a harmonic vibrating solid (or any other harmonic system) the energy $U$ can be expanded to quadratic order in the displacements $\mathbf{u}_{i}$ of the nuclei from their equilibrium positions:

$$
U=U_{\mathrm{eq}}+\sum_{i \alpha, j \beta} u_{i \alpha} \Phi_{i \alpha, j \beta} u_{j \beta},
$$

where $U_{\text {eq }}$ is the equilibrium energy, and $u_{i \alpha}$ denotes the $\alpha$ th Cartesian component of $\mathbf{u}_{i}$. In a crystal, the phonon frequencies $\omega_{\mathbf{k s}}$ (wave vector $\mathbf{k}$, branch s) are simply related to the eigenvalues of the force-constant matrix $\Phi_{i \alpha, j \beta}$. These eigenvalues are straightforwardly calculated by diagonalization of the dynamical matrix $D_{\alpha \beta}(\mathbf{k})$, which is the Fourier transform of the force-constant matrix:

$$
D_{\alpha \beta}(\mathbf{k})=\frac{1}{m} \sum_{j} \Phi_{i \alpha, j \beta} \exp \left[i \mathbf{k} \cdot\left(\mathbf{R}_{i}^{0}-\mathbf{R}_{j}^{0}\right)\right],
$$

where $\mathbf{R}_{i}^{0}$ is the position of lattice site $i$. (For ease of writing, we assume here a monatomic crystal with one atom per primitive cell.) The vibrational free energy per atom is then given by:

$$
F_{\mathrm{vib}}=\frac{1}{N_{\mathbf{k}}} \sum_{\mathbf{k} s}\left[\frac{1}{2} \hbar \omega_{\mathbf{k s}}+k_{B} T \ln \left(1-e^{-\beta \hbar \omega_{\mathbf{k s}}}\right)\right],
$$

where the sum goes over the Brillouin zone, with $N_{\mathbf{k}}$ the number of $\mathbf{k}$ vectors in this sum.

The ab initio calculation of the force-constant matrix, and hence the phonon frequencies, of crystals has been thoroughly explored in the last few years. There are two main approaches: analytic perturbation techniques based on linear response theory [26, 27] and the finite-displacement technique, based on the $a b$ initio calculation of the forces induced by chosen displacements [28, 29]. The latter technique uses the fact that the force-constant matrix gives the proportionality between the forces $F_{i \alpha}$ (atom $i$, Cartesian component $\alpha$ ) and the displacements:

$$
F_{i \alpha}=-\sum_{j \beta} \Phi_{i \alpha, j \beta} u_{j \beta} \text {. }
$$

This relation is applied directly by calculating the forces on the atoms in a large periodically repeated cell for chosen displacements. Symmetry is used to reduce to a minimum the number of independent displacements that are needed. For further details, see Ref. [28].

For anharmonic systems, and for liquids, a more powerful and general approach is needed. The strategy that has proved most successful is that of "thermodynamic integration" [30], which allows the free energy associated with the ab initio energy $U\left(\mathbf{R}_{1}, \ldots, \mathbf{R}_{N}\right)$ to be related to that of a reference system described by a simpler energy function $U_{0}\left(\mathbf{R}_{1}, \ldots, \mathbf{R}_{N}\right)$. The difference of free energy $\Delta F$ between the systems is equal to the reversible work 
done on switching the energy function isothermally from $U_{0}$ to $U$. In order to achieve this, one needs a continuously variable energy function $U_{\lambda}\left(\mathbf{R}_{1}, \ldots, \mathbf{R}_{N}\right)$, which depends on a "switching parameter" $\lambda$, defined so that $U_{\lambda}=U_{0}$ for $\lambda=0$, and $U_{\lambda}=U$ for $\lambda=1$. It is then a standard result of classical statistical mechanics that the change of Helmholtz free energy caused by the infinitesimal change $d \lambda$, this change being made at constant temperature and volume, is:

$$
d F=\left\langle d U_{\lambda} / d \lambda\right\rangle_{\lambda} d \lambda,
$$

where the thermal average $\langle\cdot\rangle_{\lambda}$ is evaluated in the canonical ensemble generated by the energy function $U_{\lambda}$. By integrating this relation, one obtains the difference of free energy between the systems $U_{0}$ and $U$ :

$$
\Delta F=\int_{0}^{1} d \lambda\left\langle d U_{\lambda} / d \lambda\right\rangle_{\lambda}
$$

In many cases, the simplest procedure is to use a linear switching between the two energy functions, defined by:

$$
U_{\lambda}=(1-\lambda) U_{0}+\lambda U,
$$

in which case

$$
\Delta F=\int_{0}^{1} d \lambda\left\langle U-U_{0}\right\rangle_{\lambda}
$$

In order to calculate the ab initio free energy by this route, one must choose a reference system whose free energy is already known or is easily calculable; in some cases, a system described by pair potentials will be suitable. The difference $\Delta F$ is then calculated by evaluating $\left\langle U-U_{0}\right\rangle_{\lambda}$ at a series of values of $\lambda$ between 0 and 1 , and performing the integral numerically. The evaluation of $\left\langle U-U_{0}\right\rangle_{\lambda}$ is done by ab initio molecular dynamics, with the energy $U_{\lambda}$ used to generate the dynamics. It should be noted that the foregoing thermodynamic integration formulas hold only for classical systems because they assume that $d U_{\lambda} / d \lambda$ commutes with the kinetic energy.

The success or failure of this whole scheme depends heavily on the choice of reference system because this determines the effort needed to compute $\Delta F$. No general prescription can be given for choosing $U_{0}$, but the crucial requirement is that the fluctuations of $U-U_{0}$ should be as small as possible in the ensemble generated by $U_{\lambda}$. There are two reasons for this. The first is that the statistical accuracy of $\left\langle U-U_{0}\right\rangle_{\lambda}$ in a run of given length is determined by the strength of the fluctuations (and, of course, by their memory time). If we require a certain precision in $\Delta F$, this fixes the length of the runs needed to achieve it. The second reason is that $\left\langle U-U_{0}\right\rangle_{\lambda}$ varies less with $\lambda$ if the fluctuations are small, so that a smaller number of $\lambda$ values suffices to do the integration. Although there are no general rules for choosing $U_{0}$, there is an obvious choice for vibrating solids, which is to choose $U_{0}$ as the harmonic part of the ab initio $U$ given in Eq. (3). The condition that quantum effects must be negligible for thermodynamic integration to be valid should not be problematic here, since anharmonicity is normally only significant at temperatures well above the Debye temperature. On the other hand, Eq. (5) for the harmonic free energy $F_{\text {vib }}$ fully includes quantum effects.

Thermodynamic integration is the method of choice for calculating the ab initio free energy of liquids. Its use for calculating the free energy of anharmonic solids will be shown in the next section.

\section{Practical Examples}

The ab initio calculation of melting properties provides an excellent illustration of the ideas we have outlined, and we shall concentrate mainly on this, but we shall also touch on the recent use of DFT to calculate the free energy of formation of lattice defects. The first fully ab initio study of melting was that of Car and Sugino [1] on silicon; more recently, we reported a detailed study of the melting properties of aluminum [2]. It is important to understand that high precision is crucial to the success of this type of calculation, for a reason that is easy to explain. The melting temperature is determined by plotting the Gibbs free energies of solid and liquid as a function of $T$ for fixed $p$, and by determining the point where the curves cross. But they cross at a shallow angle. Since the entropy $S$ is $-(\partial G / \partial T)_{p}$, the difference of slopes at the point of intersection is $\Delta S$, the entropy of fusion. If the free energy of the liquid is subject to an error $\delta G$, which is not canceled by a similar error for the solid (or vice versa), then the error in the melting temperature will be $\delta T_{m}=\delta G / \Delta S$. But since $\Delta S$ for most monatomic materials is approximately $k_{B}$ per atom (it is actually $1.4 k_{B}$ for $\mathrm{Al}$ ), then if we aim to calculate $T_{m}$ within $100 \mathrm{~K}$, the uncanceled error $\delta G$ must be no more than ca. $10 \mathrm{meV}$. This implies that the absolute technical precision for noncanceling errors of solid and liquid separately must exceed this tolerance. The question of how this can be achieved was examined 
in detail in our work on $\mathrm{Al}$ [2], and we summarize the key points here.

For liquid $\mathrm{Al}$, our ab initio simulations revealed that the Lennard-Jones model provides an excellent reference system. In this model, the total energy is a sum of pair potentials of the form

$$
\phi(r)=4 \epsilon\left[\left(\frac{\sigma}{r}\right)^{12}-\left(\frac{\sigma}{r}\right)^{6}\right],
$$

where $r$ is the interatomic distance. For each thermodynamic state, we can choose the parameters $\epsilon$ and $\sigma$ so that the structures of the ab initio liquid and the Lennard-Jones liquid are almost identical, in which case the average $\left\langle U-U_{0}\right\rangle_{\lambda}$ in the thermodynamic integral is almost independent of $\lambda$. Our tests showed that the required precision can be achieved by ignoring the variation with $\lambda$ and setting $\left\langle U-U_{0}\right\rangle_{\lambda}$ equal to its value for $\lambda=\frac{1}{2}$. The free energy of the Lennard-Jones reference liquid has been extensively investigated by other researchers, and we used the accurate parameterized form published by Johnson et al. [31].

It is important to stress that the free energy that we need to compute refers to the thermodynamic limit, in which the number of atoms in the system tends to infinity, with the density held fixed. But ab initio molecular dynamics calculations can only be done for rather modest sizes of periodically repeated cells; our ab initio simulations on liquid Al were done with systems of only 64 atoms, so finite-size errors need to be carefully considered. The crucial point here is that the Lennard-Jones free energies refer already to the thermodynamic limit, so that we are concerned only with errors for the quantity $\left\langle U-U_{0}\right\rangle_{\lambda}$, and this is much less sensitive to system size. Our tests suggest that size errors for this quantity are probably well within the required tolerance already with 64 atoms.

For the crystal, a good approximation to the free energy is obtained by ignoring anharmonic effects and basing the calculation on the harmonic phonon frequencies. Nevertheless, anharmonic corrections are not negligible, and we use thermodynamic integration to calculate them. Our ab initio calculations of the phonon frequencies $\omega_{\mathbf{k} s}$ of the face-centredcubic aluminum crystal were performed using the finite-displacement method [28] explained in the previous section. Since all atoms in a face-centredcubic crystal, and all three Cartesian directions, are equivalent by symmetry, displacement of a single atom in a single direction suffices.

Having obtained the harmonic free energy, the thermodynamic integration formula (10) is applied to obtain anharmonic corrections, with $U_{0}$ now representing the $\mathrm{ab}$ initio harmonic energy, and $U$ the full ab initio energy. Sampling over configuration space was performed by ab initio molecular dynamical (MD) simulation. Here, it is crucial to recognize that conventional dynamics suffers from a serious problem because in a harmonic system equipartition between vibrational modes is never achieved. To overcome this ergodicity bottleneck, we use instead the constant-temperature MD technique of Andersen [32], in which the atomic velocities are randomized at frequent intervals. We find that for $\mathrm{Al}$, anharmonicity raises the crystal free energy enough to lower the melting temperature by ca. $80 \mathrm{~K}$.

The melting temperature, entropy, and volume change on melting and other properties for aluminum that emerge from our calculations [2] are compared with experimental values in Table I. The comparison with experiment is very satisfying, especially when it is recalled that the final results depend on no fitting to experimental data whatever. The calculated $T_{m}$ is correct to ca. $5 \%$, and the entropy and volume of fusion are in almost perfect agreement with experimental values.

The techniques used in the earlier ab initio work of Car and Sugino [1] on the melting of Si were similar to those we have just described. The reference system used for both the liquid and the solid was the Stillinger-Weber (SW) interaction model [33], for which the free energy is accurately known. (We remark that the Lennard-Jones model would be a very poor model for Si because it gives closepacked hard-sphere-like structures for both solid and liquid, whereas crystalline $\mathrm{Si}$ is tetrahedrally

TABLE I

Comparison between experimental and calculated melting properties of Al from Ref. [2]. ${ }^{a}$

\begin{tabular}{lcl}
\hline & Experiment & Calculation \\
\hline$T_{m}(\mathrm{~K})$ & 933.47 & $890 \pm 20$ \\
$\Delta S\left(k_{B} /\right.$ atom $)$ & 1.38 & $1.36 \pm 0.04$ \\
$\Delta H(\mathrm{eV} /$ atom $)$ & 0.111 & $0.104 \pm 0.003$ \\
$\Delta V\left(\AA^{3} /\right.$ atom $)$ & 1.24 & $1.26 \pm 0.2$ \\
$d T_{m} / d P(\mathrm{~K} / \mathrm{GPa})$ & 65 & $67 \pm 12$
\end{tabular}

a Listed are the melting temperature $\left(T_{m}\right)$, the entropy of fusion $\Delta S$, the enthalpy of fusion $\Delta H$ (related to $\Delta S$ by $\Delta H=$ $T_{m} \Delta S$ ), the volume of fusion $\Delta V$, and the derivative of $T_{m}$ with respect to pressure. For sources of experimental data, see Ref. [2]. 
coordinated and liquid $\mathrm{Si}$ is approximately sixfold coordinated [34].) As in our work on Al, careful attention was paid to size effects, $k$-point sampling errors, and other technical aspects. In calculating the free energy of the crystal, Car and Sugino [1] used the SW model as reference system, whereas we would strongly advocate use of the ab initio harmonic system. However, we have no reason to suppose that their choice of reference system caused any problems.

The calculated melting properties of Si [1] are compared with experimental values in Table II. The accord with experiment is not as good as what we found for $\mathrm{Al}$, the calculated $T_{m}$ being too low by ca. $20 \%$. However, the predicted entropy and volume of fusion appear to be very satisfactory. There are reasons why Si may be more difficult than a simple metal like Al. Crystalline $\mathrm{Si}$ is a semiconductor (the experimental band-gap is $1.1 \mathrm{eV}$ ), whereas the liquid is a metal. The way in which electronic charges are screened therefore changes significantly on melting, and it may be that the LDA does not account for these changes accurately enough. Such problems are not expected in a free-electron-like metal such as Al.

Before leaving the subject of phase equilibria, we note that the ability to calculate the free energies associated with lattice vibrations opens up the possibility of calculating the relative thermodynamic stabilities of different crystal structures, and phase boundaries between them. A notable recent achievement has been the very recent work of Pavone et al. [35] on the transition between the $\alpha$ and $\beta$ phases of tin. The $\alpha$ phase is more stable at low temperatures, and the DFT-pseudopotential calcu-

\section{TABLE II}

Comparison between experimental and calculated melting properties of Si from Ref. [1]. ${ }^{a}$

\begin{tabular}{llc}
\hline & Experiment & Calculation \\
\hline$T_{m}(\mathrm{~K})$ & 1685 & 1350 \\
$\Delta S\left(k_{B} /\right.$ atom $)$ & $3.6,3.3$ & 3.0 \\
$\Delta H(\mathrm{eV} /$ atom $)$ & $0.52,0.47$ & 0.35 \\
$\Delta V\left(\AA^{3} /\right.$ atom $)$ & $-2.43,-1.94$ & -2.00 \\
$d T_{m} / d P(\mathrm{~K} / \mathrm{GPa})$ & -38 & -50 \\
\hline
\end{tabular}

a Listed are the melting temperature $\left(T_{m}\right)$, the entropy of fusion $\Delta S$, the enthalpy of fusion $\Delta H$ (related to $\Delta S$ by $\Delta H=$ $T_{m} \Delta S$ ), the volume of fusion $\Delta V$, and the derivative of $T_{m}$ with respect to pressure. For sources of experimental data, see Ref. [1]. lations confirm that at zero temperature its energy is slightly below that of the $\beta$ phase. However, the vibrational properties of the $\beta$ phase make its entropy larger at high temperatures. The calculations predict a zero-pressure transition temperature of $311 \mathrm{~K}$, which is very close to the experimental temperature of $286 \mathrm{~K}$. Other recent work on solid-state phase stability is reported in Refs. [29, 36-38]. The ab initio calculation of vibrational free energies also makes it possible to study thermal expansion [39] and other thermodynamic properties of crystals.

We now turn briefly to the ab initio treatment of lattice defects. In thermal equilibrium, all crystals contain point defects, and in most materials the vacancy is the dominant defect. (In some materials, thermally generated interstitials are also important.) At temperature $T$, the thermal equilibrium concentration $c_{\text {vac }}$ of vacancies, i.e., the mean number of vacancies per lattice site, is given by [40]:

$$
c_{\mathrm{vac}}=\exp \left(-\beta g_{\mathrm{vac}}\right) \text {, }
$$

where $g_{\mathrm{vac}}$ is the free energy of formation of a vacancy. To be more precise, $g_{\mathrm{vac}}$ is the change of Gibbs free energy of the system when an atom is removed from a specified lattice site and the removed atom is then replaced to create a new bulk lattice site, the whole process being performed reversibly and at constant pressure. Alternatively, and completely equivalently, $g_{\text {vac }}$ can be regarded as the change of Helmholtz free energy in this process, provided it is performed at constant volume.

We can express $g_{\text {vac }}$ as the sum of two terms: first, the change of free energy $g_{\text {vac }}^{(1)}$ when a chosen atom is reversibly removed from a lattice site and parked in some convenient "holding state;" second, the difference $g_{\text {vac }}^{(2)}$ between the free energy of the atom in the holding state and the free energy per atom of the perfect bulk crystal:

$$
g_{\mathrm{vac}}=g_{\mathrm{vac}}^{(1)}+g_{\mathrm{vac}}^{(2)} .
$$

The choice of holding state is arbitrary and makes no difference to the final result.

In the holding state used in Refs. [3-6], the removed atom is completely isolated from all other atoms in the system, but is acted on by a harmonicoscillator potential. With this choice, $g_{\mathrm{vac}}^{(2)}$ is straightforward to calculate: The free energy of the atom in the holding state is trivial, and the free energy per atom in the bulk crystal can be computed as explained earlier in this section. The contribution $g_{\text {vac }}^{(1)}$ is calculated by thermodynamic integration, and for this we need a continuous reversible path connecting the end states: the initial state is the perfect bulk 
crystal; the final state is the crystal containing a single vacancy, together with the isolated atom in the holding state. A path can be constructed by continuously decoupling the chosen atom from the rest of the system, while simultaneously switching in the holding potential, the point on the path being specified by the coupling parameter $\lambda$.

In practice, the decoupling process is full of interesting subtleties. Since the DFT/pseudopotential technique is used, decoupling actually means switching off the core charge of the removed ion and the pseudopotential through which it interacts with the valence electrons. This means that the atom in the holding state is actually a bare uncharged core. But the vacancy system so produced must contain $Z$ fewer valence electrons than the initial bulk system, where $Z$ is the core charge of the removed atom. It is therefore essential that $Z$ valence electrons be made to disappear in a continuous way during the decoupling process. Although electrons do not behave like Cheshire cats in the real world, their continuously variable presence fits very naturally with the conventional machinery of DFT, where partial electronic occupation numbers are routinely [41] used. For full details of these and other subtleties, we refer the reader to the original studies. As pointed out by Smargiassi and Madden [5, 6], the general principles just outlined are readily adapted to deal with other defects, such as the divacancy and interstitial.

The first practical calculations on defect concentrations using these ab initio methods were on the vacancy in crystalline Si $[3,4]$. This was followed by detailed free energy calculations on the vacancy, the divacancy, and self-interstitial in $\mathrm{Na}$, reported by Smargiassi and Madden [5, 6]. The reader is referred to the original studies for details of the results.

\section{Future Prospects}

The examples we have presented leave no doubt that the $a b$ initio calculation of free energies and other thermodynamic functions of condensed phases is now a practical possibility. To clarify what has been achieved, we return to the distinction made earlier between a fully ab initio calculation of thermodynamic functions and a calculation based on a parameterized interaction model fitted to $a b$ initio calculations. In the techniques we have outlined, interaction models play a role as reference systems in thermodynamic integration. But it is crucial to appreciate that the final results do not depend in any way on the choice of these models. This choice affects only the route by which these results are obtained. The situation is fundamentally different if thermodynamic functions are calculated solely from a fitted interaction model, because then the final results clearly do depend on the choice of model. Almost all fitting procedures involve fairly arbitrary choices, and the likely errors are hard to judge. The ability to avoid such uncertainties by performing direct $a b$ initio calculations of thermodynamic functions is therefore a significant step forward.

But, of course, uncertainties remain because DFT cannot be made exact. We stressed that in order to treat melting with useful accuracy the calculation of free energies must achieve a technical precision of ca. $10 \mathrm{meV}$ per atom. Needless to say, this is well beyond the absolute accuracy of practical DFT calculations. (For example, one would not expect to predict cohesive energies to better than a few times $100 \mathrm{meV}$.) However, the size of absolute errors is irrelevant. The relevant issue is the size of "noncanceling errors": errors that do not cancel between the two phases in equilibrium. In the melting of metals, for example, where the atomic coordination and electronic structure are often almost identical in the coexisting solid and liquid, a high degree of cancellation of DFT errors is expected, so that reduction of noncanceling errors below $10 \mathrm{meV}$ is not unrealistic. What remains is then the reduction of other noncanceling errors-those due to k-point sampling, size effects, statistical sampling, etc.-below this threshold. This is entirely achievable.

We believe that the ab initio calculation of phase equilibria and defect concentrations is potentially of great importance. One scientific area that is likely to benefit in the near future is the earth sciences. Under the conditions of extreme pressure and temperature found in planetary interiors, phase equilibria are difficult to determine experimentally, and information from ab initio calculations will be very valuable. The techniques we have outlined will be directly applicable to solid-solid and solid-liquid equilibria both in iron and in silicates, which are the main constituents of Earth's core and mantle, respectively. Defect processes play a key role in the convection of Earth's mantle, which is responsible for continental drift.

If we can calculate free energies, then it should be possible to calculate chemical potentials, and hence solubilities, and the compositions of coexisting phases in multicomponent systems. A large amount of experience in the calculation of chemical potentials has already been accumulated using sim- 
ulation methods based on classical potentials [30]. A commonly used procedure is to obtain chemical potentials by a thermodynamic integration in which one chemical species is transmuted into another. There is no doubt that such procedures could be applied to fully ab initio simulation. However, given the much greater computational effort needed in ab initio calculations, it is clear that carefully designed reference systems will play a key role here, as they have already done in melting calculations.

We conclude with some comments about the inclusion of electronic thermal excitations and nuclear quantum effects. Electronic excitations present no problem in principle, and may be important for matter under extreme conditions. Mermin's finitetemperature generalization of DFT [42] is already quite widely used in the treatment of metallic systems [41]. The key issue here is the possible temperature dependence of the exchange-correlation energy, and very little is yet known about the accuracy of finite-temperature DFT in practical calculations.

Concerning nuclear quantum effects, we have noted that the key formula of thermodynamic integration, Eq. (10), is valid only in classical statistical mechanics because it assumes that the energy $U$ commutes with the nuclear kinetic energy. However, this limitation is straightforward (though possibly expensive) to overcome. Feynman's pathintegral formulation of quantum mechanics $[43,44]$ provides the basis for simulations of systems in thermal equilibrium with full inclusion of nuclear quantum effects [45-47]. The combination of pathintegral simulation with ab initio DFT methods has already been used in practical calculations [48, 49], and the evaluation of free energies by this approach looks perfectly feasible.

\section{ACKNOWLEDGMENTS}

The work of D.A. and G.A.dW. was supported by NERC grant GST/O2/1454 to G. D. Price and M. J. Gillan. The work of G.K. was supported by EPSRC grants GR/L08946 and GR/L38592. The position of M.J.G. at UCL is financially supported by GEC. We thank the High Performance Computing Initiative for allocations of time on the Cray T3D and T3E at Edinburgh Parallel Computing Centre, these allocations being provided through the Minerals Physics Consortium (GST/O2/1002) and the U.K. Car-Parrinello Consortium.

\section{References}

1. Sugino, O.; Car, R. Phys Rev Lett 1995, 74, 1823.

2. de Wijs, G. A.; Kresse, G.; Gillan, M. J. Phys Rev B 1998, 57, 8223.

3. Car, R.; Blöchl, P.; Smargiassi, E. Mat Sci Forum 1992, 83-87, 443.

4. Smargiassi, E.; Car, R. Phys Rev B 1996, 53, 9760.

5. Smargiassi, E.; Madden, P. A. Phys Rev B 1995, 51, 117.

6. Smargiassi, E.; Madden, P. A. Phys Rev B 1995, 51, 129.

7. Parr, R. G.; Yang, W. Density-Functional Theory of Atoms and Molecules; Oxford University Press: Oxford, 1989.

8. Jones, R. O.; Gunnarsson, O. Rev Mod Phys 1989, 61, 689.

9. Seminario, J. M.; Politzer, P., Eds. Modern Density Functional Theory: A Tool for Chemistry; Elsevier: Amsterdam, 1995.

10. Hohenberg, P.; Kohn, W. Phys Rev 1964, 136, B864.

11. Kohn, W.; Sham, L. J. Phys Rev 1965, 140, A1133.

12. Perdew, J. P.; Chevary, J. A.; Vosko, S. H.; Jackson, K. A.; Pederson, M. R.; Singh, D. J.; Fiolhais, C. Phys Rev 1992, 46, 6671.

13. Ziesche, P.; Kurth, S.; Perdew, J. P. Comput Mat Sci 1998, 11, 122.

14. Tozer, D. J.; Handy, N. C. J Phys Chem A 1998, 102, 3162.

15. Heine, V. In Solid State Physics; Ehrenreich, H.; Seitz, F.; Turnbull, D., Eds.; Academic Press: New York, 1970; Vol. 24, p. 1.

16. Cohen, M.; Heine, V. In Solid State Physics, Ehrenreich, H.; Seitz, F.; Turnbull, D., Eds.; Academic Press: New York, 1970; Vol. 24, p. 38.

17. Heine, V.; Weaire, D. In Solid State Physics; Ehrenreich, H.; Seitz, F.; Turnbull, D., Eds.; Academic Press: New York, 1970; Vol. 24, p. 250.

18. Bachelet, G. B.; Hamann, D. R.; Schlüter, M. Phys Rev B 1982, 26, 4199.

19. Vanderbilt, D. Phys Rev B 1990, 41, 7892.

20. Kresse, G.; Hafner, J. J Phys Condens Mat 1994, 6, 8245.

21. Car, R.; Parrinello, M. Phys Rev Lett 1985, 55, 1471.

22. Payne, M. C.; Teter, M. P.; Allan, D. C.; Arias, T. A.; Joannopoulos, J. D. Rev Mod Phys 1992, 64, 1045.

23. Kresse, G.; Furthmüller, J. Phys Rev B 1996, 54, 11169.

24. Kresse, G.; Furthmüller, J. Comput Mat Sci 1996, 6, 15.

25. Gillan, M. J. Contemp Phys 1997, 38, 115.

26. Giannozzi, P.; de Gironcoli, S.; Pavone, P.; Baroni, S. Phys Rev B 1991, 43, 7231.

27. Dal Corso, A.; Pasquarello, A.; Baldereschi, A. Phys Rev B 1997, 56, 11369.

28. Kresse, G.; Furthmüller, J.; Hafner, J. Europhys Lett 1995, 32, 729.

29. Warren, M. C.; Ackland, G.; Karki, B. B.; Clark, S. J. Minera$\log$ Mag 1998, 62, 585.

30. Frenkel, D.; Smit, B. Understanding Molecular Simulation; Academic: San Diego, 1996.

31. Johnson, J. K.; Zollweg, J. A.; Gubbins, K. E. Molec Phy 1993, 78, 591.

32. Andersen, H. C. J Chem Phys 1980, 72, 2384. 


\section{DEVELOPMENTS IN AB INITIO THERMODYNAMICS}

33. Stillinger, F. H.; Weber, T. A. Phys Rev B 1985, 31, 5262.

34. Štich, I.; Car, R.; Parrinello, M. Phys Rev B 1991, 44, 4262.

35. Pavone, P.; Baroni, S.; de Gironcoli, S. Phys Rev B 1998, 57, 10421.

36. van de Walle, A.; Ceder, G.; Waghmare, U. V. Phys Rev Lett $1998,80,4911$.

37. Ceder, G. Current Opinion Solid State Mat Sci 1998, 3, 533.

38. Vočadlo, L.; Brodholt, J.; Alfè, D.; Gillan, M. J.; Price, G. D. Phys Earth Planet Inter 2000, 117, 123-137.

39. Lazzeri, M.; de Gironcoli, S. Phys Rev Lett 1998, 81, 2096.

40. Henderson, B. Defects in Crystalline Solids; Arnold: London, 1972.

41. Gillan, M. J. J Phys Condens Mat 1989, 1, 689.
42. Mermin, N. D. Phys Rev 1965, 137, A1441.

43. Feynman, R. P. Rev Mod Phys 1948, 20, 367.

44. Feynman, R. P.; Hibbs, A. R. Quantum Mechanics and Path Integrals; McGraw-Hill: New York, 1965.

45. Chandler, D.; Wolynes, P. G. J Chem Phys 1981, 74, 7.

46. Berne, B. J.; Thirumalai, D. Ann Rev Phys Chem 1986, 37, 401.

47. Gillan, M. J. In Computer Modelling of Fluids, Polymers and Solids; NATO ASI Series C: Mathematical and Physical Sciences, Vol. 293; Catlow, C. R. A.; Parker, S. C.; Allen, M. P., Eds.; Kluwer: Dordrecht, 1990; p. 155.

48. Benoit, M.; Marx, D.; Parrinello, M. Nature 1998, 392, 258.

49. Biermann, S.; Hohl, D.; Marx, D. J Low Temp Phys 1998, 110, 97. 